\title{
PRINCIPE MONARCHIQUE OU ROI TRÈS CHRÉTIEN? LES FUNÉRAILLES DU ROI DE FRANCE
}

Dans le sillage du grand livre d'Ernst Kantorowicz, Les Deux corps du $r o i^{1}$, une école historique, maintenant couramment désignée par l'expression : « école cérémonialiste américaine », a pris en compte les grandes cérémonies monarchiques pour la compréhension de la genèse de l'État moderne. Sacre, funérailles royales, Lits de Justice et entrées royales ne sont plus seulement envisagés d'un point de vue anecdotique, de celui de leurs fastes, ou encore sous l'angle plus large d'une incompréhensible monarchie qui finit par oublier elle-même le sens de gestes et de symboles qui ne sont plus de ce fait que les preuves d'un invraisemblable archaïsme. Au contraire, dans l'interprétation de la symbolique royale mise en cuvre dans des rituels que l'on cherche à expliquer, ces chercheurs trouvent l'essence même des principes du régime, pour tout dire, et le mot est prononcé, la constitution même de l'Ancien Régime.

Le principal mérite de cette "école cérémonialiste" est sans aucun doute d'avoir attiré l'attention des historiens sur le sens de telles cérémonies, hors des idées toutes faites sur la monarchie ou des préjugés qui courent sur elle depuis la Révolution française. Mona Ozouf et Maurice Agulhon ont bien montré, sans références particulières à ces travaux américains, que la Révolution ne s'est pas faite sans création de cérémonies nouvelles et la République sans mise en œuvre de symboles adéquats. Fêtes révolutionnaires et Mariannes participent à l'histoire du nouveau régime de la France en lui donnant sens et en forgeant dans les consciences une représentation qui se veut pertinente de ses apports spécifiques $^{2}$. L'Ancien Régime, par les cérémonies qui se tiennent autour de

\footnotetext{
1. Ernst Kantorowicz, Les Deux corps du roi. Essai sur la théologie politique au Moyen Age, Paris, Gallimard, 1989 ; la première édition, en anglais, date de 1957.

2. Mona Ozouf, La Fête révolutionnaire (1789-1799), Paris, Gallimard, 1976. Maurice Agulnon, Marianne au combat. L'imagerie et la symbolique républicaines de 1789 à 1880 , Paris, Flammarion, 1979.
}

Revue de synthèse : IV S. Nos 3-4, juil.-déc. 1991. 
la personne du roi, dont il est le centre, mais qui le mettent tout autant au centre procède de mêmes principes, de mêmes mécanismes.

Faut-il pour cela voir dans les rites et les symboles la traduction d'une constitution, qui pour n'être écrite nulle part n'en est pas moins stricte, constitution coutumière qui ne se rapprocherait pas de celle de l'Angleterre pour autant, dans la mesure où celle-ci ne s'exprime pas ou pas seulement, ou d'autre manière, dans ces rites et ces cérémonies? La distinction entre constitution coutumière et constitution écrite n'a pas seulement été utilisée pour comparer des régimes politiques contemporains l'un de l'autre, comme celui de l'Angleterre ou de la France, mais certains auteurs l'utilisent également pour distinguer les périodes et les régimes politiques dans un même pays. Maurice Duverger, qui voit dans les lois fondamentales du royaume la constitution coutumière de la France d'Ancien Régime est de ceux-là ${ }^{3}$. Les principes politiques que l'on regroupe en constitution coutumière, s'ils veulent être perçus comme ayant force de constitution, doivent sans doute se traduire d'une manière ou d'une autre dans des textes autant que dans des gestes et des symboles. Car il n'y a au fond de constitutions qu'écrites. C'est par commodité de classement que l'on parle de constitution coutumière pour exposer les principes et les règles qui, ailleurs ou autrefois, régissent ou régissaient la vie politique avec la même force qu'une constitution. Quand, au XVIII siècle, le mot constitution apparaît, il est le plus souvent employé métaphoriquement : "la constitution du royaume " est à entendre comme la constitution d'une personne, robuste ou faible. L'usage qui est le nôtre en dérive chez les penseurs des Lumières, mais dans ce sens précisément; Turgot rappelle à Louis XVI que son " royaume n'a pas de constitution ".

Dans sa « Harangue aux états généraux de 1561 », le chancelier Michel de l'Hospital distingue clairement les lois qui « sont les fondements des républiques » des autres qui "dépendent de la grâce et bienfait du prince ". Mais cette vision duale de la loi, qui est à l'origine de la notion de lois fondamentales du royaume élaborée par les juristes et les historiens, ne permet pas de parler de constitution, même coutumière. S'il est vrai que ces lois sont destinées à assurer l'unité du royaume - on les nomme : "lois de l'État du royaume " au xvĩ siècle - elles ne soumettent pas toutes les autres lois édictées par le prince à une dépendance hiérarchique, mais définissent deux champs distincts de la loi. De ce fait, d'ailleurs, elles ne permettent pas de définir une quelconque limitation de

3. Maurice Duverger, Les Constitutions de la France, Paris, Presses universitaires de France, 1944, $8^{\mathrm{e}}$ éd. 1964 : introd. et chap. 1 dont le titre est : « L'Ancien Régime : dix siècles de stabilité constitutionnelle », p. 9 à 37 . 
pouvoir, et Robert Descimon, s'appuyant sur les théoriciens du temps, ramène ces lois fondamentales à la seule loi de dévolution de la couronne : la loi salique ${ }^{4}$. Et quand bien même les principes fondamentaux de la monarchie française s'expriment publiquement avec faste et grandeur dans les cérémonies royales, ils se dégagent tout autant d'un ensemble de textes, de problèmes plus ou moins réglés par les cours souveraines, de situations qui ont fait naître toutes sortes de décisions, et tout cela, consigné dans des écrits variés, ne forme pas, au sens strict, une constitution. Pour autant, l'Ancien Régime n'est pas sans principes, sans institutions réglées et sans lois; il est même le moment où, sous la forme de l'État, prend forme l'arsenal d'idées et de principes, d'institutions et de lois, où la politique puise sa première modernité.

Des quatre grandes cérémonies royales - le sacre, les funérailles, les entrées et les Lits de Justice ${ }^{5}-$ il en est une qui illustre bien les résultats obtenus par les cérémonialistes héritiers de Kantorowicz : les funérailles royales, étudiées par son élève Ralph Giesey. Funérailles royales et sacre sont deux cérémonies étroitement liées. L'une comme l'autre marquent le passage d'un roi à un autre et assurent donc la continuité de la monarchie. Dès 1142 , la chancellerie royale prend l'habitude de dater le début d'un règne du jour du décès du roi précédent. Quand saint Louis meurt en 1270 près de Tunis, son fils Philippe III qui se trouve près de lui est proclamé immédiatement roi par l'entourage royal. Avant son sacre, il assume tous les pouvoirs politiques d'un roi de France. Tous ses successeurs suivent son exemple et de ce fait l'exercice du pouvoir monarchique précède la cérémonie d'investiture. Le sacre perd alors toute valeur légale ; au plan du droit, il n'est plus la cérémonie constitutive d'un règne. Les funérailles royales tendent à le remplacer dans ce rôle et prennent à la fin du Moyen Âge une importance de plus en plus grande qui culmine au $x v^{e}$ siècle. C'est alors la cérémonie royale la plus longue. Elle tombe en désuétude $\mathrm{au} \mathrm{xvII}^{\mathrm{e}}$ siècle. Les dernières grandes funérailles royales sont

4. Voir Robert Descimon, « L'Unité du droit public : loi fondamentale et limitation du pouvoir ", in Robert DESCIMON et Alain GuERY, " Un État des Temps Modernes? », dans L'État et les pouvoirs de l'Histoire de la France, publiée par André Burgulère et Jacques Revel, Paris, Seuil, 1990, p. 219-232.

5. Les grands livres de "l'école cérémonialiste américaine » qui en traitent sont les suivants : Ralph E. GIESEY, Le Roi ne meurt jamais. Les obsèques royales dans la France de la Renaissance, Paris, Flammarion, 1987; Richard A. JACKSON, Vivat Rex. Histoire des sacres et couronnements en France, 1364-1825, Strasbourg, Association des publications près les universités, 1984 ; Sarah Hanley, Le Lit de Justice des rois de France. L'idéologie constitutionnelle dans la légende, le rituel et le discours, Paris, Aubier, 1991 ; Lawrence M. BRYANT, The King and the City in the Parisian Royal Entry Ceremony. Politics, Ritual, and Art in the Renaissance, Genève, Droz, 1986. R. E. GiEsEY a rédigé une petite synthèse qui tient compte de ses nouvelles recherches, sous le titre : Cérémonial et puissance souveraine - France, $x v^{2}$ xutf siècle, Paris, A. Colin, 1987. 
celles d'Henri IV en 1610. C'est à ce moment, on l'a vu, que le sacre se charge d'un sens nouveau, après les modifications apportées dans la seconde moitié du $\mathrm{Xvi}^{\mathrm{e}}$ siècle.

Le déroulement des funérailles des rois de France est bien connu dans ses principaux moments. Après un service mortuaire à Notre-Dame de Paris, le roi défunt est emmené à l'abbaye de Saint-Denis, par un trajet qui reprend en partie celui de l'entrée solennelle du roi dans sa capitale, après son sacre. Là, les serviteurs de sa maison lancent leurs bâtons dans la tombe royale et la bannière de France est abaissée sur le cercueil au cri de : « Le roi est mort », pour être presque immédiatement relevée au cri de : "Vive le roi ». Aux insignes royaux qui sont placés sur le cercueil du roi défunt succède en 1422 une effigie de Charles VI, revêtue de tous ces insignes. Cette effigie est primitivement destinée à remplacer le corps du roi mort sur son lit de parade, au-delà du moment où il ne serait plus en état d'être exposé. L'effigie du roi défunt assiste aux repas de cérémonie. Elle est menée en procession. Et ce personnage de cire, paré de tous les ornements de la royauté, contraste avec le corps réel du roi défunt, qu'on ne voit plus. L'effigie ressemble plus au roi, tel qu'on l'a vu ou dans l'idée qu'on s'en fait, qu'à celui qui est mené au tombeau. En 1498, le Parlement de Paris peut faire évoluer sans difficulté majeure la signification de cette représentation réaliste du roi. Il décide que l'effigie représente le roi vivant et non le roi mort. Seul un être vivant peut participer à des banquets, à des processions. L'usage de masques mortuaires pour fabriquer les effigies, la représentation des yeux ouverts sur les visages de cire, à partir de 1498, simulent un être vivant. Aux funérailles de Louis XII en 1515 , l'effigie royale n'est plus placée au-dessus du cercueil du roi. Elle le suit, portée en triomphe sous le dais. Lors des funérailles de François $\mathrm{I}^{\mathrm{er}}$, l'effigie royale est exposée d'abord, elle assiste à un banquet où un repas lui est servi, dans un décor aux couleurs vives. Dans la même salle, le cercueil remplace ensuite l'effigie, les tentures noires les tissus chamarés. Ce roi "vivant » en effigie a posé un problème concernant l'assistance de son fils aux funérailles du roi défunt. Car si le règne commence à la date même de la mort du roi précédent, la monarchie dépassant la personne mortelle des rois et étant éternelle, l'héritier du trône est déjà roi quand l'effigie de son père met en face de lui un autre roi « vivant ». Pour éviter une confusion bien compréhensible, on demande au nouveau roi de ne pas assister aux funérailles du roi précédent. Le dédoublement de la cérémonie lors des funérailles de François $\mathrm{I}^{\text {er }}$ permet de mettre en évidence le principe des deux corps du roi, l'un symbolisant la monarchie, à qui l'on rend hommage, avant l'autre, simple homme décédé, à qui est rendu un service funèbre religieux. Néanmoins, Henri II n'accompagne pas le cortège aux funérailles de son père. Caché, il le regarde passer derrière une 
fenêtre. Il ne peut, il ne doit, en toutes circonstances, y avoir qu'un seul roi de France.

Paradoxalement, cette mise à l'écart du nouveau roi lors des funérailles de son père, si nécessaire qu'elle ait pu apparaître pour montrer que si le roi meurt, la monarchie qu'il incarne ne meurt pas avec lui, est sans doute une des causes de la désuétude dans laquelle sont tombées les funérailles royales aux $\mathrm{XVII}^{\mathrm{e}}$ et $\mathrm{XvIII}^{e}$ siècles. Nécessairement absent de cette cérémonie dont la signification est capitale pour le pouvoir qu'il incarne, il ne peut se " rattraper ", pourrait-on dire, qu'avec son sacre. Aussi, celui-ci devient-il de plus en plus important pour les rois, dès la seconde moitié du $x^{e}{ }^{e}$ siècle. Il finit par l'emporter, aux $x^{\prime} I^{e}$ et $x v I^{e}$ siècles sur les funérailles royales. Après les funérailles d'Henri IV, en 1610, où pour la dernière fois on utilise l'effigie du roi défunt, donc la fiction des deux corps du roi, l'un immortel symbolisant la monarchie, l'autre mortel, celui de l'homme qui a rempli temporairement la fonction de roi, c'est la métaphore du soleil, déjà introduite dans le rituel du sacre, avec le lever du roi dormant, qui est chargée de faire sentir et comprendre lors des funérailles royales, que la monarchie ne meurt pas en France.

Toute l'interprétation de Ralph E. Giesey est donc fondée sur la présence d'une effigie représentant le roi vivant et parée des atours de sa fonction lors de ses funérailles. Effigie et représentation sont des mots employés dans les sens que nous leur connaissons encore, dès le Moyen Âge. Seul, le verbe « effigier » n'est plus usité. Dans les rites et cérémonies funèbres, effigie et représentation ont une signification plus particulière. Jean Nicot, dans son Thrésor de la langue françoyse tant ancienne que moderne, publié pour la première fois en 1606, mentionne " une représentation de bière que l'on met au service d'un trépassé » et donne comme équivalent d'une telle représentation: cenotaphium, tumulus honorarium. Furetière précise que le mot «se dit aussi à l'Église d'un faux cercueil de bois couvert d'un poêle de deuil, autour duquel on allume des cierges lorsqu'on fait un service pour un mort ». " La représentation est l'image qui nous remet en idée et en la mémoire des objets absents, et qui nous les peint tels qu'ils sont ", ajoute-t-il ; «Quand on va voir les Princes morts dans leur lit de parade, on n'en voit que la représentation, l'effigie ". Et à ce dernier mot, Furetière revient sur l'équivalence : effigie $=$ représentation en redonnant le même cas d'espèce : "On voit les rois en effigie dans leur lit de parade " et en citant un exemple d'effigie, celle du roi Henri IV qui est visible dans le trésor de Saint-Denis. Le dictionnaire de l'Académie française, dans sa première édition de 1694, précise :

«Après la mort des rois et des grands Princes, on expose leur effigie en public. C'est-à-dire, leur représentation en cire. On sert leur effigie pendant 
quelques jours avec les mesmes cérémonies qu'on a accoustumé d'observer pour leur propre personne."

Les textes dont nous disposons sur les rites funéraires des rois n'ont pas la précision chronologique que Giesey a déterminée pour l'utilisation d'une effigie (de Charles VI à Henri IV, de 1422 à 1610). Des textes des $\mathrm{XV}^{\mathrm{e}}$ et $\mathrm{XVI}^{\mathrm{e}}$ siècles passent rapidement sur l'effigie, quand d'autres des $\mathrm{XvII}^{\mathrm{e}}$ et $\mathrm{XVIII}^{\mathrm{e}}$ siècles insistent au contraire sur cette pratique. Nicot, au début du XviI ${ }^{\mathrm{e}}$ siècle ne mentionne pas l'emploi particulier d'une effigie royale lors des funérailles du monarque, alors que Furetière et l'Académie française insistent au contraire sur ce cas, à la fin du Xvir siècle. Par contre, tous les ouvrages mentionnent l'effigie parmi d'autres formes de représentation possibles. Selon eux, elle s'intègre aux décorations funèbres au même titre que les statues, les bustes, les tableaux, qui jouent dans les cérémonies le même rôle. Les cénotaphes, lors des cérémonies religieuses, symbolisent le corps du défunt lorsqu'il n'est pas présent. Ils prennent la forme d'un cercueil recouvert d'un grand poêle, usage commun pour les messes des morts, d'un tombeau honoraire et temporaire, ou d'une effigie au naturel couchée sur un lit, nous dit le père Menestrier, auteur d'un ouvrage de synthèse sur « les décorations funèbres » publié en 1683.

Pour cet auteur, introducteur de la mode italienne en France dans ce domaine particulier, les effigies restent des représentations possibles :

«On met [dans les chapelles ardentes] la figure de personnes pour qui se font les funérailles, ou en portrait, ou en médaille, ou en statue, ou en buste, ou en image de cire d'après le naturel, vêtue et couchée sur un lit ${ }^{6}$.

Mais il renvoie cette pratique à une mode du passé, succédané de l'exposition du corps embaumé :

«On peut au lieu des statues et des portraits se servir des représentations au naturel avec des images de cire vêtues à la manière des personnes vivantes. On l'a souvent fait pour nos Rois dans leurs convois funèbres; et comme on est obligé d'exposer durant plusieurs jours les Princes et les Souverains sur leurs lits de parade, on se sert de ces images; parce qu'il serait difficile de conserver si longtemps des corps sans qu'ils se gatassent, quelque soin que l'on prenne de les embaumer, n'y ayant rien qui change si tôt que le visage ${ }^{7}$.

6. Père Claude François Menestrier, Des Décorations funebbres, Paris, R.J.B. de Caille, 1683 , p. 296. Sur la carrière du père Menestrier et l'influence italienne qu'il propage, voir : Paul Allut, Recherches sur la vie et les ouvrages du père Claude François Menestrier, Lyon, N. Schevring, 1856.

7. C. F. MENESTRIER, op. cit. supra n. 6, p. 204. 
Selon les auteurs des $\mathrm{XVII}^{\mathrm{e}}$ et $\mathrm{XVIII}^{\mathrm{e}}$ siècles écrivant sur les funérailles, la mode, si l'on peut dire ainsi, seule, a changé. L'usage de décorer les églises de sculptures et de peintures, pour les funérailles royales et princières, est une tradition tardive en France, qui ne s'introduit pas, venant d'Italie, avant les années 1670 . Encore en 1666, quand Charles Vigarani, officier de la Maison royale avec le titre d'inventeur des machines des théâtres et ballets de Sa Majesté rend compte à un correspondant de Modène de la pompe funèbre d'Anne d'Autriche à Saint-Denis, il est frappé du peu d'éclat de la décoration, bien inférieure à ce qu'il avait coutume de voir dans son pays d'origine, l'Italie. On s'est contenté de revêtir la cathédrale de simples tentures noires couvertes de cartouches d'armoiries et d'élever une chapelle ardente au centre du chœur ${ }^{8}$. L'Italie est alors "en avance " sur le reste de l'Europe en ce domaine. Non seulement les artistes dessinent les décors funéraires et participent à l'élaboration de la décoration, mais ces œuvres éphémères sont jugées dignes d'être reproduites et décrites par des dessins, ce qui n'est pas encore le cas en France.

C'est en août 1670 que Henri de Gissey change pour la première fois l'ordonnance habituelle des décors pour les cérémonies funèbres du duc de Beaufort et d'Henriette d'Angleterre. Désormais, les grands de ce monde sont célébrés lors de leurs funérailles au milieu de pilastres, de frontons, de statues, de tableaux, de bas-reliefs et de devises qui s'ajoutent aux tentures masquant l'ordre gothique, alors peu apprécié, de l'architecture de la cathédrale. Ce décor a un double but : augmenter le faste des cérémonies religieuses dans la cathédrale, en se conformant au goût esthétique du moment qui a changé. Le décor à la fois renforce l'aspect chrétien de la cérémonie, aux dépens de son aspect monarchique, dont seul le faste rend désormais compte, et masque un ancien ordre des choses qu'exprime l'architecture gothique dans son système même.

De ce changement, nous avons des exemples nombreux. Ainsi, Le Brun est appelé à l'organisation du décor funèbre des funérailles du chancelier Séguier en 1672. S'il reprend la manière fastueuse des Italiens, il n'en conserve pas pour autant tous les éléments. Car la cérémonie ayant lieu à l'Oratoire, Le Brun ne se préoccupe pas d'en masquer l'architecture mais au contraire il cherche à tirer parti des éléments architecturaux " modernes ", qu'il se contente de souligner. Menestrier confirme bien la mise en cause de l'architecture gothique en soulignant dans son ouvrage que si la manière de Le Brun est la meilleure, elle n'est valable que dans

8. André Tessier, "Le genre décoratif funèbre - Esquisse d'une histoire de ses débuts en France ", Revue de l'art ancien et moderne, t. XLVI, 1924, p. 275. 
les églises d'architecture classique, et non dans celles qui sont antérieures ${ }^{9}$. Le Brun, a contrario, démontre le changement dans les goûts. Mais ce changement manifeste aussi une clarification des significations. Un des thèmes constamment représenté lors des funérailles des grands, et des membres de la famille royale en particulier, est celui de la victoire de l'immortalité sur la mort. Menestrier rend compte ainsi de la cérémonie des funérailles du cardinal Mazarin, dans l'église des Saints Vincent et Anastase à Rome :

"On avait mis sur la porte un grand trophée de la mort, qui fouloit aux pieds la Thiare, les Chapeaux de Cardinaux, les Sceptres, les Couronnes et les autres marques d'honneur. Deux autres morts estoient couchés sur le fronton de la porte, et entre les colonnes estoient d'un costé un tableau de l'action qu'il fit à Casa empêchant les deux armées de se battre avec cette inscription : "Pugnandum Compositor"; Et de l'autre costé la Paix de Saint Jean de Luz, avec ces mots : "Regnum Conciliator" " 10 .

Immortalité dans les consciences, pour l'action passée, pour les actes et non pour les vains symboles du pouvoir. Les funérailles de Turenne, que Louis XIV fait ensevelir en 1675 parmi les sépultures royales de SaintDenis, ont été organisées par le père Menestrier lui-même. Un Turenne victorieux est représenté sur une image portée par l'immortalité foulant

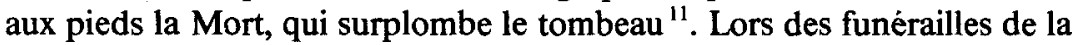
reine Marie-Thérèse, en 1683, Berain a fait placer au-dessus du jubé, visà-vis de l'autel, un grand cartouche aux armes de la reine, entouré de têtes de morts, de sceptres brisés et de couronnes renversées. Sur l'autre face, dans la nef, un bas-relief feint de marbre blanc représente la reine sortant du tombeau, accompagnée de deux anges qui la mènent au ciel, tandis qu'à ses pieds, la mort est déconfite ${ }^{12}$. Toutes les représentations hésitent entre les thèmes de la vanité, alors fréquents dans la peinture, et ceux complémentaires de l'immortalité de l'âme du chrétien.

L'effigie, qu'elle soit de cire ou de marbre, en relief ou en « plate peinture ", est toujours associée au thème de l'immortalité, immortalité dans la mémoire de ceux qui restent, immortalité du chrétien surtout, qui le libère de son corps comme elle le libère de l'exercice du pouvoir. Ralph Giesey a relégué cette interprétation chrétienne des représentations par effigies à un rang secondaire, critiquant même les témoignages du temps, explicites là-dessus, alors qu'ils disent si peu pour étayer son interprétation. Ainsi Jean Du Tillet, accusé par Giesey de passer «à côté de

9. C. F. MENESTRIER, op. cit. supra n. 6, p. 149.

10. Ibid., p. $169-170$.

11. A. Tessier, art. cit. supra n. 8, p. 377.

12. Ibid., p. 181 . 
l'essentiel " ${ }^{13}$, explique la séparation du corps et de l'effigie du roi par un désir plus explicite de concentrer sur cette dernière l'idée d'immortalité : «Par ce moyen à l'effigie seule ont depuis esté rendus les honneurs appartenants au corps mis en arrière : combien que par la future résurrection il sera immortel $"{ }^{14}$. On peut discuter les thèses de Du Tillet sur bien des points, mais on ne voit pas que l'on puisse ainsi considérer comme non essentielle la thèse qu'il se contente de reprendre : l'expression de l'immortalité du chrétien qui est associée aux représentations, effigies comprises, durant toute l'histoire du christianisme. Si ces représentations sont devenues plus explicitement chrétiennes, aux xvi ${ }^{e}$ et xvir siècles, puis plus abstraites depuis le $\mathrm{xIx}^{\mathrm{e}}$ siècle, c'est qu'elles rendent compte aussi d'une conception de la mort en général, et de la mort des grands de ce monde en particulier, qui a changé.

L'effigie du roi fait partie des effigies indiscrètes : " Image si semblable à la personne qu'on ne sçauroit discerner l'image d'avec la personne" ", écrit Jean Nicot. En cela, elles le représentent, dans tous les sens du mot. Les effigies ont été créées dans un but pratique : remplacer le corps réel du roi, lorsque celui-ci n'est plus présentable. La période qui va du constat de décès aux obsèques définitives est traditionnellement très longue pour les hauts personnages dans la plupart des civilisations. Certains peuples ont adopté la pratique des sépultures provisoires, soit en exposant le corps dans des endroits appropriés, soit en l'enterrant provisoirement. Les tribus indiennes suivent la première pratique en Amérique du Nord, la seconde en Amérique du Sud ${ }^{15}$. À cette acceptation de la corruption du corps avant la cérémonie funèbre terminale, d'autres peuples, à l'inverse, opposent l'embaumement, qui doit retenir le phénomène, le repousser le plus loin possible dans le temps, ou bien même son exact contraire, la réduction totale et immédiate du corps en cendres par la crémation. L'embaumement est la pratique choisie pour conserver le corps du roi de France. Ambroise Paré critique la technique employée, qui selon lui ne permet pas une conservation convenable au-delà de la semaine, et la juge bien en retard sur celle des Égyptiens de l'Antiquité ${ }^{16}$. Dom Poirier, savant bénédictin chargé de l'exhumation des corps royaux à Saint-Denis en 1793, confirme les constats d'Ambroise Paré. Mais la

13. R. E. GIESEY, op. cit. supra n. 5, p. 190.

14. Jean Du Tillet, Recueil des Roys de France, Paris, P. Mettayer, 1607, p. 341. Ce texte date en fait de 1566; il est repris, mot pour mot par Claude GuicHARD, pour clore son livre sur les Funérailles et diverses manières d'ensevelir les Romains, Grecs et autres nations, tant anciennes que modernes, Lyon, Jean de Tournes, 1581, p. 542. Ce livre est dédié à Charles Emmanuel de Savoie.

15. Robert HeRtz, « Contribution à une étude sur la représentation collective de la mort », L'Année sociologique, 1905-1906, p. 66-67.

16. Ambroise PARE, CEuvres complètes, Paris, Malguigne, 1841, t. III, p. 478-479. 
lecture de son rapport ${ }^{17}$ montre que malgré tout, l'embaumement a fait des progrès aux $x^{e} I^{e}$ et $x v i I^{e}$ siècles.

L'effigie du roi apparaît au moment même où il est nécessaire de mettre son corps dans le cercueil de plomb. L'embaumement, toujours pratiqué, n'est pas mentionné par tous les textes, en particulier par ceux qui insistent sur l'effigie. En fait, à ce niveau, l'effigie est un équivalent plus efficace de l'embaumement. Celui-ci est une pratique courante aux $\mathrm{XIV}^{e}$ et $X V^{e}$ siècles pour les personnages importants et pas seulement pour le roi. De la même façon, l'effigie n'est pas utilisée pour représenter les traits du seul roi ${ }^{18}$. Nombre de grands seigneurs l'ont utilisée, à partir du $x v^{e}$ siècle. Ralph Giesey a rendu célèbre la cérémonie funèbre lors de laquelle on sert à l'effigie de François $\mathrm{I}^{\text {er }}$ les repas habituels, avec les honneurs dus au roi vivant ${ }^{19}$. Mais une telle cérémonie a existé aussi pour les funérailles d'Anne de Bretagne ${ }^{20}$. Et l'effigie de François $1^{\text {er }}$ est entourée de celles de ses deux fils, à Notre-Dame-de-Paris, lors de funérailles communes. Les grands personnages, par la reprise de l'effigie, cherchent-ils à imiter le roi de France, qui l'utilise depuis les funérailles de Charles VI en 1422 ? À partir des années 1470 , les ouvrages sur les rites funéraires et les pompes funèbres montrent une volonté de codifier les usages, en puisant dans les coutumes des peuples et l'histoire des modèles et des références. Aussi, il est difficile de cerner quelle est la part de l'imitation des grands et, bien sûr, du grand parmi les grands, le roi, et quelle est la part d'une croyance que l'on retrouve chez la plupart des peuples, et qui est encore vive au Moyen Âge, que tant que la décomposition du corps n'est pas avancée, la mort n'est pas totale, ce qui se traduit dans le rituel funèbre par le souci de repousser le phénomène et de ménager des étapes successives entre la vie et la mort.

Cette croyance ne devrait pas faire sourire en cette fin du $x x^{e}$ siècle. Nos techniques permettent de faire revenir à la vie, in extremis, des personnes dont les témoignages irréfutables et convergents portent d'abord sur la décorporation qu'ils ont ressentie et dont ils gardent la mémoire. Retenir la vie, freiner l'inéluctable, sont de toutes époques, mais selon des principes et des moyens qui changent dans l'histoire ${ }^{21}$. À cela peut

17. Publié par Alain Boureau, Le Simple corps du roi, Paris, Éd. de Paris, 1988, Annexe I, p. 71-90.

18. Colette Beaune, "Mourir noblement à la fin du Moyen Âge ", in La Mort au Moyen Age, Colloque de l'Association des historiens médiévistes français, Strasbourg, 1975, Strasbourg, Istra, 1977, p. 125-143.

19. R. Giesey, op. cit. supra n. 5, chap. 1, "Les funérailles de François ${ }^{\text {er }}$, p. 13-37, en part., p. 19-20.

20. Père Bernard de Monifaucon, Monuments de la monarchie française, Paris, J. M. Gandouin et P. F. Giffart, 1731, t. 3, p. 133.

21. La mort est ressentie comme un danger plus que comme un scandale. La décomposition du cadavre est donc une menace. Le rituel de la mort a donc à la fois pour but de protéger les vivants, de les rassurer, et d'assurer à celui qui meurt un statut favorable. 
s'ajouter le problème posé dans toute société par la mort du chef, du roi. Pour conjurer le trouble qui saisirait cette société, la coutume s'est installée dans beaucoup d'entre elles de cacher la mort du souverain autant que possible durant la période qui va du décès proprement dit à la décomposition du corps, garantie de sa mort certaine. On doit attendre que le roi soit complètement mort avant de pouvoir crier : "Vive le roi!» C'est bien ce qui se produit lors du rite funéraire du roi de France, déclaré effectivement mort seulement au terme de ses funérailles, des semaines après le constat de décès, tel que nous l'entendons au moment de l'ensevelissement dans le tombeau à Saint-Denis. Et c'est là qu'immédiatement après est nommé son successeur. De ce point de vue, l'effigie du roi de France, comme celle des reines, princes et autres grands seigneurs, correspond à l'idée que le roi, ce roi-là qui est représenté, est encore vivant, quand son cercueil dit qu'il est déjà mort. C'est bien de la même personne qu'il s'agit et du même corps. L'effigie prend la place du corps, embaumé ou non, qui, les textes donnent maints exemples aux xiII et XIv siècles, a été promené, exposé, dans les cortèges funèbres. Elle permet d'allonger le temps durant lequel les funérailles se déroulent, le temps qui va des derniers moments de vie du roi à son acte de décès officiel prononcé dans la crypte de Saint-Denis, qui autorise la désignation de son successeur.

Les funérailles royales sont d'abord et avant tout les funérailles d'un roi chrétien. L'embaumement puis l'effigie, ou toute autre représentation, jouent un rôle de transition, entre le moment du décès, et la mort solennellement déclarée du roi. Ralph Giesey fait de ce moment celui de « la fiction de la souveraineté post mortem » du roi, dont les robes rouges des Parlementaires, les bâtons des officiers de sa maison, et le triomphe de l'entrée du convoi dans Paris, sont autant de signes connexes ${ }^{22}$. Mais le déroulement de la cérémonie signifie d'abord que le roi, qui n'a pas encore été déclaré mort, est considéré comme toujours vivant. C'est du roi qu'on emmène là, et non de la monarchie, qu'il s'agit. L'effigie qui le montre paré de tous les atours royaux a une double signification au moins. Celle que privilégie Giesey, d'un sens exclusivement monarchique, peut très bien se voir adjoindre une signification purement religieuse. C'est bien ce qui se passe quand François Clouet, qui confectionne l'impressionnante effigie de François $I^{\text {er }}$, prévoit deux paires de mains amovibles, que l'on peut donc changer, selon les circonstances de son exposition. L'une des paires figure des mains jointes, et l'autre, des mains en position de préhension, donc pouvant recevoir le sceptre et la

22. R. GIESEY, op. cit. supra n. 5, p. 223. 
main de justice ${ }^{23}$. Par cet appareil, on peut passer facilement du symbole de l'immortalité du roi très chrétien, à l'immortalité du roi représentant la monarchie avec ses symboles. C'est alors que les auteurs qui traitent des funérailles royales commencent à donner à l'effigie un sens exclusivement religieux.

Cérémonie conforme aux croyances de la mort passage, par étapes successives, vers un autre monde, cérémonie trop exclusivement monarchique par son dispositif et ses symboles, les funérailles royales ont été rechristianisées entre le $\mathrm{XVI}^{\mathrm{e}}$ et le XvIII siècle. Les fastueuses pompes funèbres du $\mathrm{Xv}^{e}$ siècle ont laissé la place à des cérémonies plus sobres au xvi 's siècle. Saint-Simon raconte qu'il a proposé au duc d'Orléans de réduire considérablement la pompe des funérailles de Louis XIV, sur le modèle de celles de Louis XIII " qui avait tout défendu et réduit au plus simple ${ }^{24}$. Le duc d'Orléans s'y conforme, même si le décor de la cathédrale rend compte de l'importance du personnage. Cette nouvelle cérémonie n'est plus vraiment une cérémonie monarchique, mais les funérailles chrétiennes d'un roi. L'importance de la cérémonie dans la rue est réduite, au profit de l'importance de la cérémonie dans la cathédrale. Les funérailles royales sont ainsi devenues la cérémonie privée de la première famille chrétienne de France, celle du roi. Le symbolisme qui valorise la foi chrétienne prend le pas sur le symbolisme royal. Cette re-christianisation d'une cérémonie royale aboutit au paradoxe apparent de désacraliser le roi. Le roi très chrétien, comme tout chrétien, est tenu de dire vrai, et de dire vrai à propos de lui-même. Alors se pose pour lui le dilemme pascalien du roi chrétien. Peut-on agir en roi, si l'on est authentiquement chrétien? L'histoire n'a répondu qu'à la question inverse, par la négative.

\section{Alain GuÉRY, Centre de recherches historiques, Paris, C.N.R.S./E.H.E.S.S.}

23. Etienne Moreau-Nelaton, Les Clouets, peintres officiels des rois de France, Paris, H. LAURENS, 1908, t. I, p. 18-28.

24. Louis de Rouvroy, duc de SAInt-Simon, Mémoires, t. $12: 1715-1716$, Paris, Ramsay, 1978, p. 75 . 